\title{
Formulation of Stress (Strain)-induced Diffusion of Hydrogen and Its Solution by Computer-aided Finite Element Method*
}

\author{
By Shin-ichiro OCHIAI,** Shoichi YOSHINAGA** and Yoneo KIKUTA ${ }^{* *}$
}

\begin{abstract}
Synopsis
Our previous paper clarified the hydrogen-dislocation interaction and its role in hydrogen embrittlement. Now the consequence of the hydrogendislocation interaction was sought to explain stress (strain)-induced diffusion of hydrogen, assuming that the region of higher dislocation density could hold a higher hydrogen concentration per unit volume.

We have formulated the stress (strain)-induced diffusion in the form of augmented Fick's second law. Here, the local plastic strain represents the dislocation density gradient, and is coupled with the ordinary diffusion equation. The augmented equation was proved to be a successful mean to calculate, in quantitative manner, hydrogen concentration at plastically deformed notch region.

The compatibility of the calculations and experiments favours our present formulation. Thus, usually unpredictable hydrogen cracking of welded structures or steel structures in general could be predicted, provided that the equation with the corresponding boundary and initial conditions is solved successfully. Since local plastic strain is in the equation, elastic-plastic analysis is done before diffusion problem analysis. Computer-aided finite element method is the most suitable to solve such problems.
\end{abstract}

\section{Introduction}

High strength steels are in demand increasingly in accordance with more frequent use of large-scale welded structures. However they sometimes encounter sudden and unexpected crackings of the welded parts and quite often the total fracture of the structure themselves. This is often the result of hydrogen embrittlement. The high sensitivity of the above mentioned steels to the embrittlement is responsible for the calamities. Extensive researches including those of Troiano ${ }^{1)}$ have already been done. The main characterization of the embrittlement is that hydrogen gathers at defects or the region of stress triaxiality, and causes the subsequent lowering of the local material strength. However, the vagueness in the expressions and the absence of quantitative discussions are the main difficulties still to be overcome.

We have found the parallelism of hydrogen embrittlement with hydrogen-dislocation interaction as was reported in the earlier issue of present transactions. ${ }^{2)}$ Here the notch-tensile-strength of thermally hydrogenated steel*** (similar to hydrogen introduction during arc welding) decreases most when hydrogen-dislocation interaction in the specimens is the maximum. When there exist some kinds of notches in the specimens or structures, there occur stress concentration and the subsequent yielding. Such thermally hydrogenated steels undergo the dislocation multiplication. The dislocation density gradient there causes a high hydrogen concentration due to the above mentioned hydrogen-dislocation interaction. We will show how this hydrogen gathering at notch root can be formulated in the form of augmented form of ordinary Fick's second equation where local plastic strain $\bar{\varepsilon}_{p}$ will be coupled with the equation.

The elastic-plastic analysis of notched specimen is carried out to obtain $\bar{\varepsilon}_{p}$ distribution, which in turn is fed to our formulation. Both elastic-plastic analysis and diffusional analysis are executed by computer aided finite element method.

\section{Formulation of Present Stress(Strain)-in- duced Diffusion of Hydrogen}

Many discussions on stress-induced diffusion of hydrogen have been done by assuming elastic strain interaction energy between negative hydrostatic stress, i.e., $-1 / 3\left(\sigma_{x x}+\sigma_{y y}+\sigma_{z z}\right)$ and the volume change produced in lattice, i.e., $\Delta v$. Liu $^{3)}$ derived solute concentration: $C$ at crack tip for steady state diffusion under both concentration and stress gradients:

$$
C=C_{0} \exp \left[\frac{8 \pi(1+\nu) r^{3} K}{3 \sqrt{ } 2 \pi k T \sqrt{ } r_{0}}\right]
$$

where, $\quad r_{0}$ : radius of solute atom

$K:$ stress intensity factor

$r$ : radial distance from crack tip.

Using Eq. (1) St. John and Gerberich ${ }^{4)}$ estimated critical hydrogen concentration for crack growth initiation as $\simeq 7.2 \mathrm{ppm}$ for their AISI 4340 steel. However Eq. (1) can not be applied for actual case of plastically deformed crack tip. Moreover the solute atom flow could not be expected to be in a steady state in general (maybe so with stress corrosion cracking where hydrogen is continuously introduced into material from outside). The estimation of hydrogen volume, $4 \pi r_{0}^{3}$, in iron lattice is also very difficult and their conclusions are dubious. All these considerations suggest that the concentration of hydrogen gathering at crack tip can not be so high as $2 C_{0}\left(C_{0}\right.$ is hydrogen concentration far from the tip) in St. John and Gerberich's case, when only the elastic strain is taken into account as the driving force for hydrogen gathering. All the experimental results so far including those of Troiano revealed that the delayed cracking only occurs at applied stress levels where local plastic deformation (more than $0.2 \%$ plastic strain) exists around notches.

Our preceding paper showed clearly the process that hydrogen precipitates on dislocations. A dislocation (both edge and screw) interacts with impurity

* $\quad$ Received on June 28, 1974

** Department of Welding Engineering, Faculty of Engineering, Osaka University, Yamada-kami, Suita 565.

*** Rapid strain-hardening occurs for such specimens in contrast to very small strain-hardening of as-received high strength steels. 
atom $^{5)}$ (hydrogen in our case) by way of dilatation and shear, and attracts impurity to its core, and the dislocation atmosphere is formed.* According to Ref. 5 the saturation concentration near such dislocation is as high as $6 \mathrm{at} \%$ for carbon as impurity.

At equilibrium, impurity atoms are very much localized at dislocation sites (the local equilibrium between dislocation sites and lattice sites is realized soon for hydrogen due to the large value of the lattice diffusion coefficient). Thus usual use of volume as to represent sites for impurity atoms' habitation is not realistic, and rather, the dislocation length should be a proper quantity to represent the sites. Thus, we introduce a new quantity which we term as the effective concentration, $C^{*}$ which is expressed as

$$
C^{*} \equiv \frac{\mathcal{N}}{L}
$$

in contrast to the usual definition of volume concentration, $C=\mathcal{N} / V$. Thus the physical meaning of $C^{*}$ is the number of impurity atoms per unit length of dislocation.

$$
L=A V
$$

where, $A$ is local dislocation density. $C^{*}$ is now related to ordinary volume concentration $C$ :

$$
C^{*}=\frac{\mathcal{N}}{A V}=\frac{C}{A}
$$

Further, the impurity flux $J$ is assumed to be caused by the gradient of the new quantity $C^{*}$ and not by ordinary volume concentration gradient;

$$
J \equiv-\gamma D \frac{\partial C^{*}}{\partial x}
$$

where, $D$ is diffusion coefficient of the impurity atom, $\gamma$ is proportionality constant. Then the equivalence to Fick's second law can be derived from conservation of matter.

$$
\frac{\partial C}{\partial t}=\gamma D \frac{\partial^{2} C^{*}}{\partial x^{2}}
$$

For three dimensional problem, Eq. (6) is extended as:

$$
\frac{\partial C}{\partial t}=\gamma D \nabla^{2} C^{*}
$$

or

$$
\frac{\partial C^{*}(x, y, z, t)}{\partial t}=\frac{\gamma D}{\Lambda(x, y, z)} \nabla^{2} C^{*}(x, y, z, t) \ldots
$$

for the isotropic case with $C^{*}=C / \Lambda$.

Solving Eq. (8) for $C^{*}$, the ordinary volume concentration $C(x, y, z, t)$ can be readily obtained.

\section{Computer-aided Analysis of the Newly For- mulated Stress (Strain)-induced Diffusion}

To construct Eq. (8), one has to specify local dis- location density $A$. In actuality this is not easy, and we employed local equivalent plastic strain $\bar{\varepsilon}_{p}$ for the purpose. Here we assumed that the dislocation density $A$ is, in the first order of approximation, related to the plastic strain $\bar{\varepsilon}_{p}$ as follows:

$$
\Lambda=\alpha \bar{\varepsilon}_{p}(x, y, z)+\beta
$$

where, plastic strain distribution can be obtained from elastic-plastic analysis with experimentally obtained stress-strain curve of the material used. With the plastic strain distribution calculated under a specified loading condition, the diffusional problem was solved with several boundary conditions: insulating boundary, radiating boundary, and constant concentration boundary.

1. Present Tro-dimensional Elastic-plastic Analysis and the Subsequent Diffusional Problem

First, the plane stress problem was chosen for its simplicity both in elastic-plastic analysis and diffusional problem. The problem was executed by a computer-aided finite element method (Fig. 1). The matrix representation of the diffusional problem was done by using variational technique with functional in the form similar to previous workers. ${ }^{7)}$

$$
\begin{aligned}
\chi= & \iint\left[\frac{1}{2}\left\{D\left(\frac{\partial C^{*}}{\partial x}\right)^{2}+D\left(\frac{\partial C^{*}}{\partial y}\right)^{2}\right\}\right. \\
& \left.+\frac{A}{\gamma} \cdot \frac{\partial C^{*}}{\partial t} \cdot C^{*}\right] d x d y \ldots \ldots \ldots \ldots \ldots
\end{aligned}
$$

\section{START}

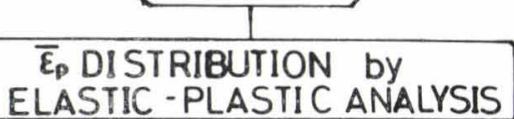
ELASTIC - PLASTIC ANALYSIS

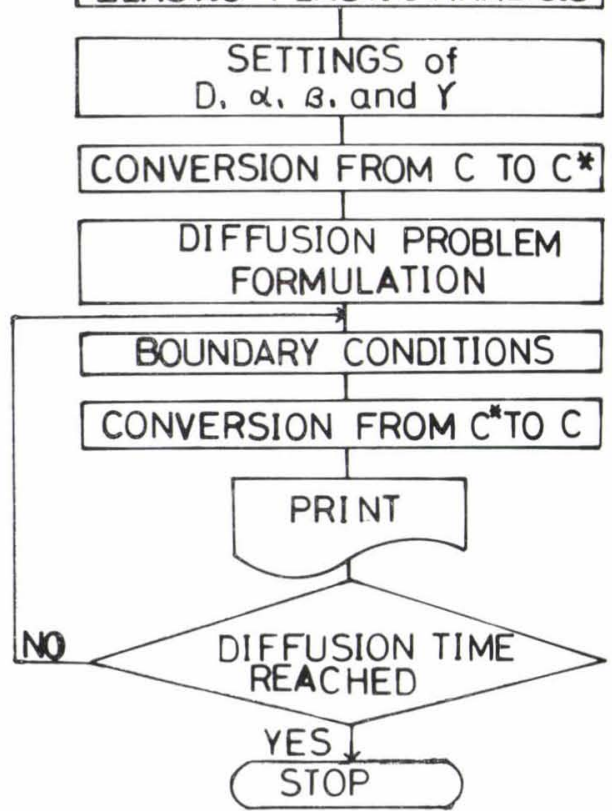

Fig. 1. Flow chart for finite element method analysis of elastic-plastic problem and hydrogen diffusional problem

\footnotetext{
* Usually the dislocation atmosphere calculation is done by using stress field of the dislocation without the atmosphere, and no allowance is made for how the strains introduced by the atmosphere affect the atmosphere and hence again the atmosphere. Such self-consistent dislocation atmosphere was recently calculated by Larché and Cahn. ${ }^{6}$
} 
Here $A / \gamma$ is taken as constant within each mesh element.

The present analysis of the newly formulated stress (strain)-induced diffusion will be correlated with the following actual delayed fracture curves in the following section. The specimen shape used for the present 2-dimensional problem is shown in Fig. 2. The specimen material is commercial HT-60 high strength steel ( $\simeq 67 \mathrm{~kg} / \mathrm{mm}^{2}$ tensile strength) with chemical composition shown in Fig. 2. Hydrogen was thermally charged and thus initial hydrogen distribution is even. Actual delayed fracture curves shown in Fig. 3 were obtained for the 2-dimensional specimens.

\section{Results by Computer}

The finite element method was applied with the mesh geometry shown in Fig. 4 together with the stress-strain curve which had been experimentally obtained as in Fig. 5.

Four different parameters $(\alpha, \beta, \gamma$ and $D)$ in Eqs. (8) and (9) were chosen as:

$\alpha=10^{14} \mathrm{l} / \mathrm{cm}^{2}, \beta=\gamma=10^{10} \mathrm{l} / \mathrm{cm}^{2}$ and $D=5 \times 10^{-7} \mathrm{~cm}^{2} /$ sec. $\beta$ was so chosen assuming that starting dislocation density (no plastic deformation) is of that order of magnitude. $\gamma$ was given the above value so that ordinary Fick's second law is deduced from Eq. (8) when there exists no plastic strain distribution. $\alpha$ was assumed to be in such an order of magnitude, considering dislocation density would increase to the order of $10^{12}$ after some percent of plastic deformation.

Typical hydrogen distribution contours were calculated and are shown in Fig. 6, where evenly distributed hydrogen re-distributes itself at notch root as

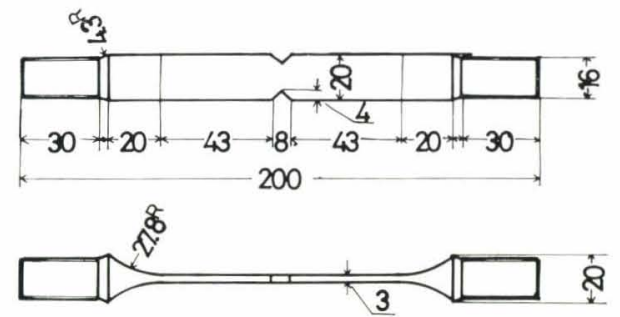

Fig. 2. Specimen dimensions (HT-60 steel); chemical composition: $\mathrm{C}\left(0.18 w \mathrm{t}^{\mathrm{o}} \%\right), \mathrm{Si}(0.55 \mathrm{wt} \%), \mathrm{P}(0.035$ $w \mathrm{t} \%), \mathrm{Ni}(0.60 \mathrm{wt} \%), \mathrm{Mo}(0.20 \mathrm{wt} \%), \mathrm{V}(0.15 \mathrm{wt} \%)$

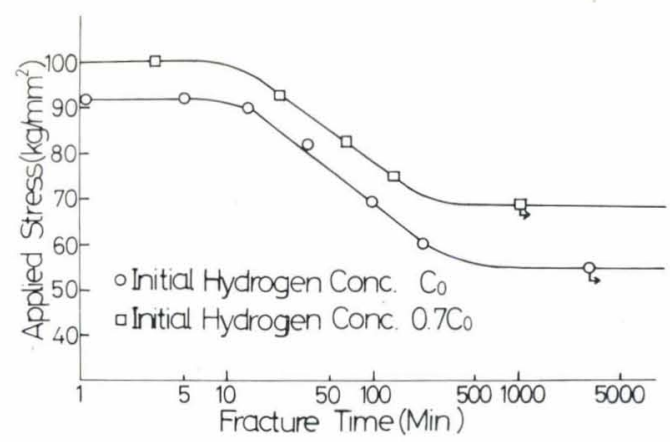

Fig. 3. Experimental delayed fracture curves for thermally hydrogen charged specimens to different initial hydrogen levels

time operates according to Eq. (8) under insulating boundary condition. Figure 7 is the similar result but with radiating boundary. In this case, the location of the highest hydrogen concentration $\left(\simeq 5 C_{0}{ }^{*}\right)$ is somewhat inside from the surface. Since the latter boundary condition is more close to reality, hydrogen, in actuality, gathers at the highly plastically deformed notch root but somewhat inside from the most severely deformed notch surface.

\section{LOAD}

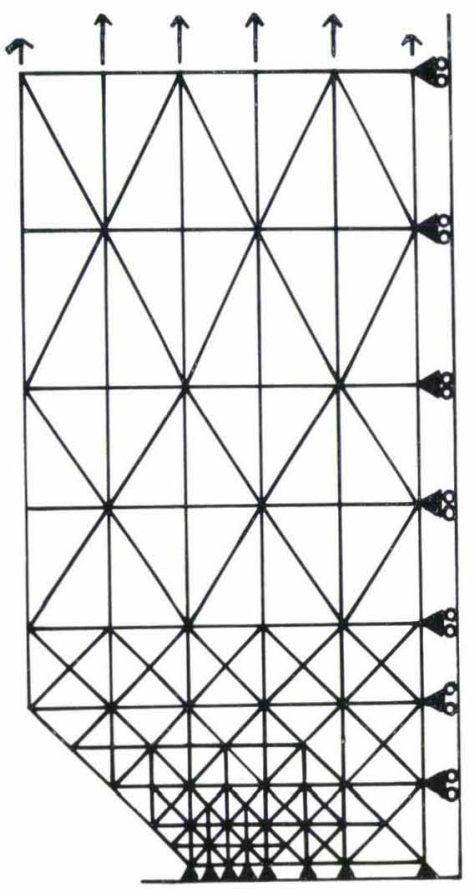

Fig. 4. Finite element mesh geometry of present notched specimen ( $1 / 4$ region): 123 elements with boundary conditions for elastic-plastic analysis.

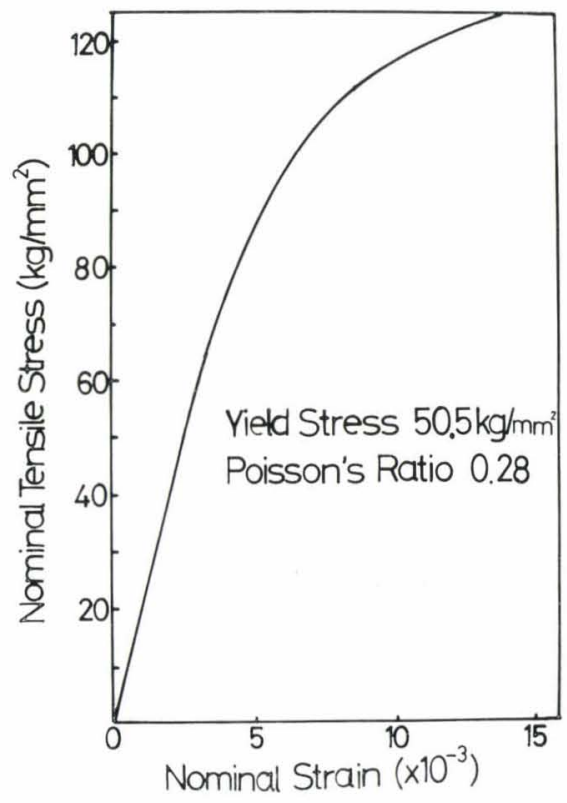

Fig. 5. Stress-strain curve of present hydrogen treated HT60 steel: $50.5 \mathrm{~kg} / \mathrm{mm}^{2}$ was chosen as a departure point from lineality.

* The actual value for $C_{0}$ is about 4.4 weight ppm. 
In hydrogen delayed cracking, hydrogen gathers at notch root gradually as time passes from the moment of loading. Thus the specimen is increasingly endangered to hydrogen-induced-cracking. Figure 8 shows such time-wise change in hydrogen concentration at notch root under insulating boundary condition. The curve with open circles and the one with closed circles correspond to different levels of initial hydrogen concentration. Both of them increase gradually as in Fig. 8 till the local concentration is sufficient to initiate crack $\left(C=C_{\mathrm{cr}}\right.$ at $\left.t=t_{\mathrm{Fer}}\right)$.

Correlation with actual delayed cracking test (Fig. 3) will lead to crack initiation at around $C \simeq 6.5 C_{0}$ of hydrogen concentration under the applied net stress of $82.14 \mathrm{~kg} / \mathrm{mm}^{2}$. Once this critical hydrogen concentration is fixed, fracture time of the specimen with a different initial concentration, but under the same loading level, can be calculated graphically on Fig. 8. For instance, when a specimen with initial hydrogen content of $C=0.7 C_{0}$ is tested under the same applied load, the delayed cracking should occur at somewhat

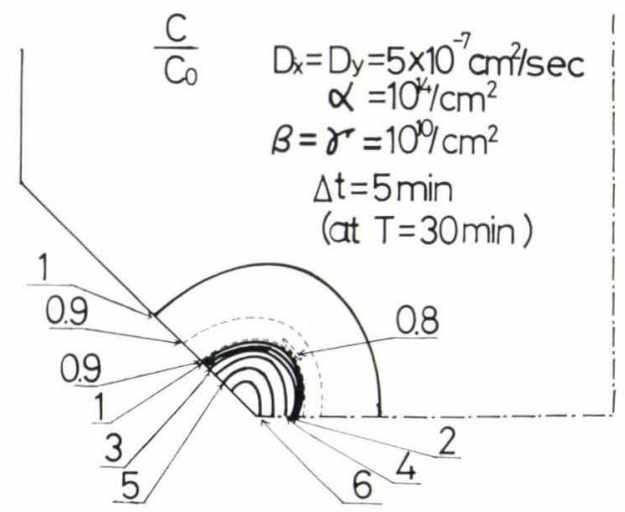

Fig. 6. Hydrogen gathering at plastically deformed notch root: Computer calculation was executed assuming insulating boundary. This shows how hydrogen redistributes itself when thermally hydrogen charged specimen was stress-aged (applied net stress of 82.14 $\mathrm{kg} / \mathrm{mm}^{2}$ ) for $30 \mathrm{~min}$.

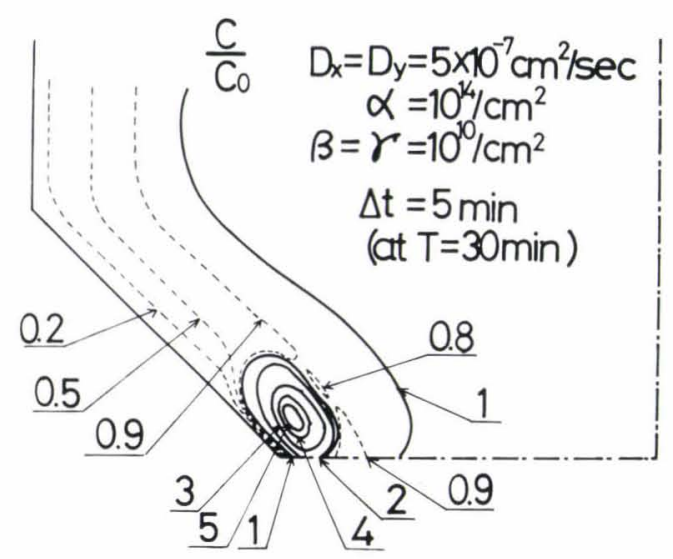

Fig. 7. Hydrogen gathering at plastically deformed notch root: Computer calculation was done assuming radiating $(C=0)$ boundary condition. This shows how hydrogen re-distribution occurs when thermally hydrogen charged specimen is stress-aged (applied net stress of $82.14 \mathrm{~kg} / \mathrm{mm}^{2}$ ) for $30 \mathrm{~min}$. longer time $\left(t_{\mathrm{Fer}}\right.$, in Fig. 8) than the corresponding value $\left(t_{\mathrm{Fcr}}\right)$ for the preceding case. The calculated value agrees well with the actual fracture time in Fig. 3 . Figure 9 is the similar curves for hydrogen timedependent gathering but under a lower applied stress level $\left(69.26 \mathrm{~kg} / \mathrm{mm}^{2}\right)$. The lower applied stress induces less hydrogen gathering while the more hydrogen gathering is now necessitated to initiate crack. Thus specimens with initial hydrogen concentration of $C=$ $C_{0}$ will undergo delayed cracking as in Fig. 9, but the ones with initial hydrogen concentration of $C=0.7 C_{0}$ would not undergo delayed cracking since hydrogen gathering at notch root does not reach the corresponding critical hydrogen concentration. This is exactly the case in actuality (Fig. 3), and the compatibility of calculations and experiments is favorable to our formulation.

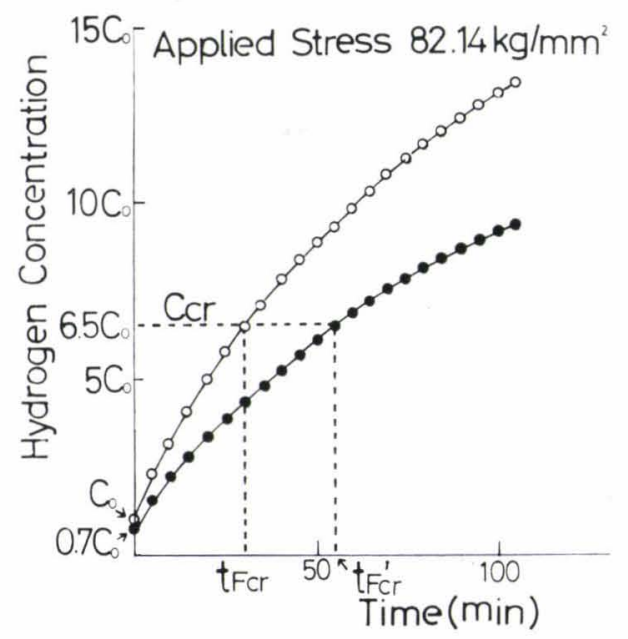

Fig. 8. Time-dependent change in hydrogen concentration at most severely plastic deformed notch root: Open circles correspond to the case where initial hydrogen occlusion was attained by hydrogenation under 1 atm of hydrogen pressure, and the closed circles for hydrogenation under 0.5 atm of hydrogen partial pressure. We can determine critical hydrogen concentration $C_{\mathrm{er}}$ from experimental fracture time $t_{\mathrm{Fer}}$ and vice versa using these curves.

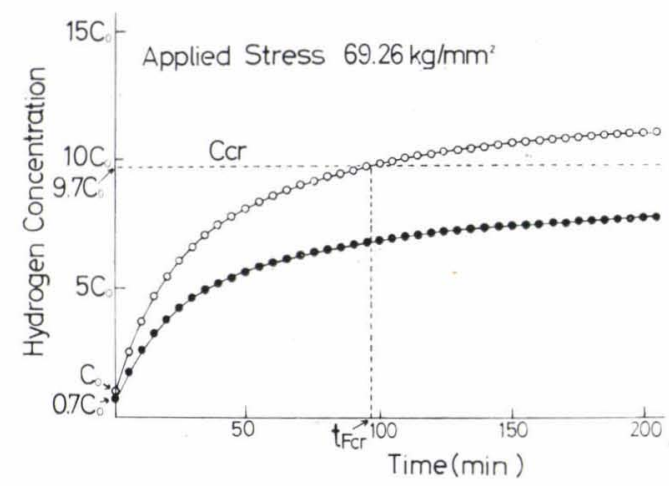

Fig. 9. Time-dependent change in hydrogen concentration at most severely plastic deformed notch root: Open circles and closed ones correspond to different initial concentration of $C_{0}$ and $0.7 C_{0}$ respectively. Critical hydrogen concentration at the level of applied stress can not be attained by closed circles. 


\section{Discussions}

Our formulation, Eq. (8), is derived from the assumption that hydrogen flux is caused not by ordinary volume concentration gradient, but by our newly defined effective hydrogen concentration $\left(C^{*}\right)$ gradient. However we have not given any physical description how impurity atom diffuses in the region which contains both dislocation sites and lattice interstitial sites. This is a very general case of diffusional problem where impurity atom diffuses over the site of different potential fields. And the chemical potential is not only the function of concentration, but also of other factors like strain energy around dislocation. To tuckle such problem correctly is almost impossible at our present expertise on diffusion problem. Even the very simple case of diffusional problem of impurity atoms around a single dislocation is only recently solved properly by Larché and Cahn. ${ }^{6)}$

Rather we use analogy discussion between diffusion and heat flow to back up our formulation (Eq. (8)). In the problem of heat flow, the equation to work with is as follows:

$$
a(x, y, z) \frac{\partial \theta}{\partial t}=K \nabla^{2} \theta \quad \text { with } \quad \delta \mathrm{Q}=a \delta \theta \quad \ldots . .
$$

where, $a$ : heat capacity per unit volume in $\mathrm{cal} \cdot \mathrm{cm}^{-3}$. $\operatorname{deg}^{-1}$

$K$ : thermal conductivity in $\mathrm{cal} \cdot \mathrm{cm}^{-1} \cdot \mathrm{sec}^{-1}$. $\operatorname{deg}^{-1}$

$\theta:$ temperature in deg

Q: heat per unit volume in $\mathrm{cal} \cdot \mathrm{cm}^{-3}$.

To see the analogy, we show ordinary Fick's second law:

$$
\frac{\partial C}{\partial t}=D \nabla^{2} C
$$

The basic difference between the two is that heat is transfered in the former case as in contrast to mass which is transfered in the latter. Another difference would be that the factor corresponding to the above mentioned heat capacity (i.e., a) is missing in the ordinary diffusional equation. On the other hand, our formulation (Eq. (8)) can be rewritten as:

$$
\frac{A}{\gamma} \frac{\partial C^{*}}{\partial t}=D \nabla^{2} C^{*} \quad \text { with } \quad C^{*}=\frac{C}{A}
$$

Now we see a clear analogy between our diffusional formulation and heat flow equation. The analogies are:

$$
a \longleftrightarrow \frac{A}{\gamma}, \quad \theta \longleftrightarrow C^{*} \quad \text { and } \quad \mathrm{Q} \longleftrightarrow \frac{C}{\gamma}
$$

Probably the better analogies can be seen by redefining $C^{*} \equiv C /(\Lambda / \gamma)$ with the analogies, i.e., $a \leftrightarrow \Lambda / \gamma$, $\theta \leftrightarrow C^{*}$ and $\mathrm{Q} \leftrightarrow C$. The reason for the better analogies is that $A / \gamma$ is indicative of relative easiness for impurity habitation (more dislocation sites), and $\mathrm{Q}$ (or $C$ ) is heat (or number of impurity atoms) per unit volume in accordance with the physical meanings of heat and mass transfer.

Microscopically hydrogen diffuses toward dislocation sites along potential gradient as well as toward less populated volume region by way of concentration gradient. For such problem, completely sound picture and its formulation into the equation comparable to Fick's second law are almost impossible to attain. Our picture and its formulation are still assumptive ones, which are dared because of our previous result on dislocation-hydrogen interaction and the following logic that the region of higher dislocation density can hold higher hydrogen concentration per unit volume.

\section{Conclusions}

We have formulated stress (strain)-induced diffusion in the form of augmented Fick's second law. Here local plastic strain $\bar{\varepsilon}_{p}$ is coupled with ordinary diffusion equation. The augmented equation was proved to be a successful mean to calculate, in a quantitative manner, hydrogen concentration at plastically deformed notch region.

Unpredictable hydrogen cracking of welded structures or steel structures in general could be predicted if the equation with the corresponding boundary and initial conditions were solved successfully. Since local plastic strain is in the equation, elastic-plastic analysis must be done before diffusional problem. The computer-aided finite element method is the most suitable method to solve such problems.

\section{Acknowledgements}

The authors gratefully acknowledge the Sakko-kai Foundation for its financial support.

\section{REFERENCES}

1) A. R. Troiano: Trans. ASM, 52 (1960), 54

2) Y. Kikuta, K. Sugimoto, S. I. Ochiai and K. Iwata: Trans. ISIJ, 15 (1975), 87.

3) H. W. Liu: ASME, J. Basic Eng., 92 (1970), 633.

4) C. St. John and W. W. Gerberich: Met. Trans., 4 (1973), 589.

5) A. W. Cochardt, G. Schoek and H. Wiedersich: Acta Met., 3 (1955), 533

6) F. Larché and J. W. Cahn: Acta Met., 21 (1973), 1051.

7) K. Honda, T. Kihara, T. Watanabe, T. Kawai, Y. Furutani and S. Shiina: Monthly J. Inst. Indust. Sci., Univ. Tokyo (Seisan-Kenkyu), 24 (1972), 44. 